\title{
An Inversion of Acoustical Attenuation Measurements to Deduce Bubble Populations
}

\author{
H. CZERSKI \\ Institute of Sound and Vibration Research, University of Southampton, Highfield, Southampton, United Kingdom
}

(Manuscript received 16 September 2011, in final form 3 February 2012)

\begin{abstract}
Measurement of natural bubble populations is required for many areas of ocean science. Acoustical methods have considerable potential for achieving this goal because bubbles scatter sound strongly close to their natural frequency, which depends largely on the bubble radius. The principle of using bulk acoustical attenuation caused by a bubble population to infer the number and size of bubbles present is well established, and appropriate methods for measuring broadband acoustical attenuation are also well developed. However, the numerical methods currently used to invert the acoustical attenuation to get the bubble size distribution are complex and time consuming. In this paper, a method for the inversion is presented that uses the physics of bubble resonance to restructure the problem so that it can be accurately carried out using a simple matrix inversion. This inversion method produces results that are correct to within a few percent over two orders of magnitude of bubble size. The most significant remaining issue for acoustical bubble measurement is the potential presence of bubbles that are resonant outside the measurement frequency range. The mathematical structure outlined here considerably simplifies the investigation of this problem, and calculations are presented that show this effect to be minor in many cases.
\end{abstract}

\section{Introduction}

The presence of bubbles in a liquid can have a considerable influence on that liquid's optical and acoustical properties, as well as providing opportunities for the exchange of gases across the bubble boundary. Consequently, accurate measurement of the number and size of the bubbles present is desirable in many fields of science, for example, chemical engineering, medicine, and oceanography. The aim of the research presented in this paper is to improve the measurement of the natural bubble populations in the ocean, and it was motivated by the increasing interest in measuring bubbles with radii less than $50 \mu \mathrm{m}$. However, the principle described may be useful in other applications as well.

It is now four decades since Medwin (1970) introduced the concept of measuring oceanic bubble populations by using the bulk acoustical properties of the bubbly water to infer the bubble size distribution present. The main advantages of the technique are that the instrumentation is relatively simple, nonintrusive, and robust enough to be

Corresponding author address: H. Czerski, Institute for Sound and Vibration Research, Highfield, University of Southampton, SO17 1BJ, United Kingdom.

E-mail: h.czerski@soton.ac.uk deployed in rough conditions at sea. In addition, measurements can be made continuously at $1 \mathrm{~Hz}$ or faster, which is sufficient to follow the evolution of bubble clouds from a breaking wave once the initial intense period of turbulence and very high void fraction is over. Over the length of time that this technique has been in use, there have been several proposed methods for inverting the acoustical attenuation to infer the bubble population present. The reason for this is that even though the contribution to acoustical attenuation made by a single size of bubble is greatest at its natural frequency, the effects at other frequencies may still be significant. This complicates the calculation of the bubble size distribution, and to date a universally satisfactory method for carrying out the inversion has not been found.

Medwin (1977) and Breitz and Medwin (1989) laid the theoretical foundations for the inversion process. Two equivalent sets of units are used in the literature for acoustical attenuation: decibels, which are commonly used by acousticians, and a pressure amplitude attenuation coefficient $b$ in nepers per meter, which is more commonly used by physicists. Here we will use $b$ because it is simpler to express, but both will be set out below for clarity. The total acoustical power attenuation $\alpha_{\mathrm{dB}}$ in decibels per meter at a given frequency $f$ is expressed as 

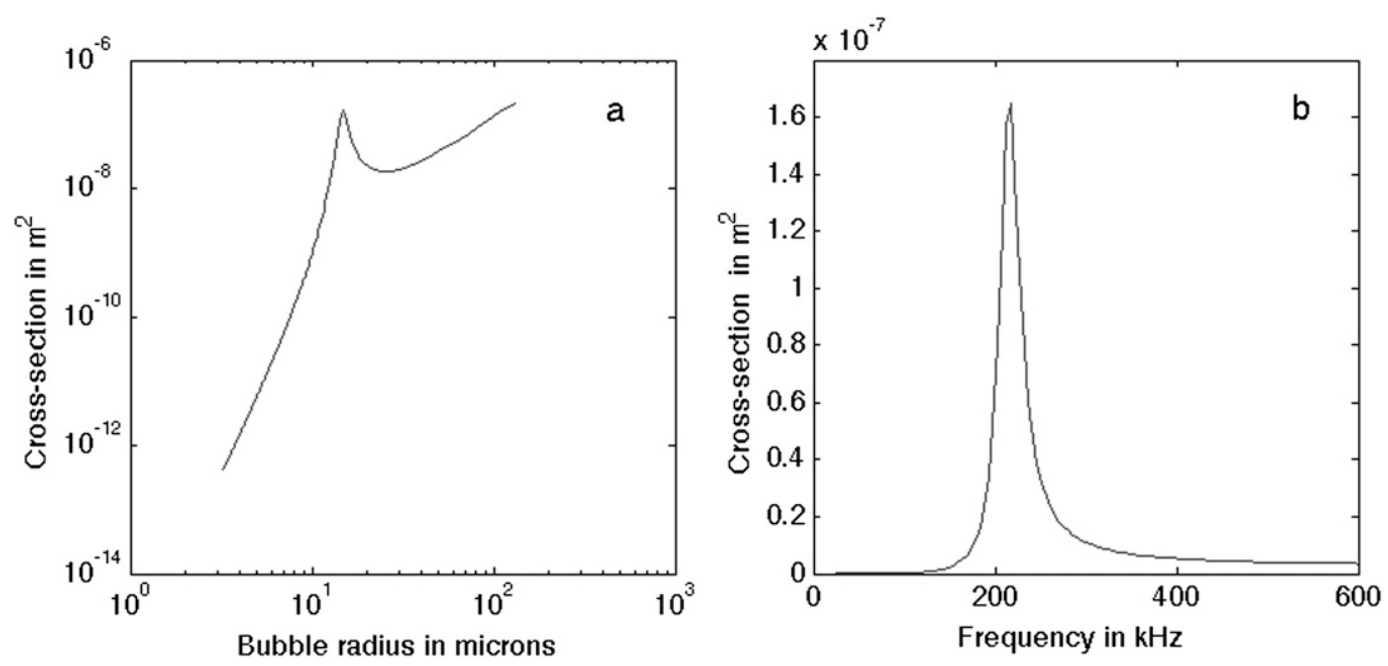

FIG. 1. Extinction cross sections: (a) variation in cross section with radius for $217 \mathrm{kHz}$, which is the resonant frequency for a $15-\mu \mathrm{m}$ bubble, and (b) variation with frequency for a single bubble with a radius of $15 \mu \mathrm{m}$.

$$
\alpha_{\mathrm{dB}}(f)=4.34 \int_{0}^{\infty} \sigma_{\text {ext }}(f, a) n(a) d a,
$$

where $n(a) d a$ is the number of bubbles with radii between $a$ and $a+d a$ per unit volume. The factor of 4.34 comes from the conversion to decibels (Medwin 2005; Leighton 1994, p. 28), where $I=I_{0} 10^{-\left(\alpha_{\mathrm{dB}} x / 10\right)}$, where $I_{0}$ and $I$ are the initial and final intensities and $x$ is the distance traveled. For calculations using the construction $I=I_{0} e^{-\alpha x}$, this factor is not needed, and the attenuation referred to in this paper will be calculated in this way. In these units, the power attenuation coefficient $\alpha$ is given by $\alpha=2 b$, so that the correct version of Eq. (1) for our purposes is

$$
\alpha(f)=\int_{0}^{\infty} \sigma_{\text {ext }}(f, a) n(a) d a .
$$

The extinction cross section $\sigma_{\text {ext }}\left(\omega, a_{0}\right)$ resulting from a bubble of radius $a_{0}$ at angular frequency $\omega$ (where $\omega=$ $2 \pi f$ ) is given by Eqs. (4.27) and (4.31) in Leighton (1994) as

$$
\sigma_{\text {ext }}\left(\omega, a_{0}\right)=\frac{d_{\text {tot }}}{d_{\text {rad }}} \frac{4 \pi a_{0}^{2}}{\left[\left(\frac{\omega_{0}}{\omega}\right)^{2}-1\right]^{2}+\left(\frac{2 \beta_{\text {tot }}}{\omega}\right)^{2}},
$$

where $d_{\text {tot }}$ is the total damping constant, $d_{\text {rad }}$ is the radiation damping constant, $a_{0}$ is the bubble equilibrium radius, $\omega_{0}$ is its resonant frequency, $\omega$ is the frequency of interest, and $\beta_{\text {tot }}$ is the total resistive constant leading to damping. Equation (2) is a complete description of the acoustical extinction and includes both resonant and geometric effects. Geometric scattering is that due to the purely cross-sectional area of the bubble, separate from any resonant effects, and it increases as $a_{0}^{2}$. In theory, if the attenuation with frequency is known, Eqs. (1) and
(2) could be inverted to calculate $n(a) d a$. Consequently, a single broadband measurement could be used to infer the bubble population over a wide radius range.

Figure 1 shows two aspects of the extinction cross section: the variation with radius at a single frequency $(217 \mathrm{kHz})$ and the variation with frequency for a bubble of fixed radius $(15 \mu \mathrm{m})$. The steadily rising slope on the right of the resonant peak in Fig. 1a represents geometric scattering, which is the acoustic scattering resulting from the physical size of a bubble (Leighton 1994). The difficulty that this geometric scattering causes is that at a single relatively high frequency, attenuation can potentially be due to either small resonant bubbles or the geometric scattering (effectively the acoustic shadow) from a range of much larger bubbles. Figure $1 \mathrm{~b}$ illustrates that a bubble of a single size causes attenuation over a narrow range of frequencies, not just its resonant frequency. Here, this range is referred to as the bubble bandwidth. A solution to Eqs. (1) and (2) must take both of these features into account.

A solution to first order was derived by Medwin (1977), by assuming that the measured attenuation was dominated by resonant scattering only (commonly referred to as the resonant bubble approximation). A simplified expression was derived that directly related measured attenuation to the number of resonant bubbles present. The limitations of this approach were investigated by Commander and Moritz (1989), and they concluded that the off-resonant contributions to scattering needed to be included in future inversion methods.

Several methods have been proposed to take the off-resonant contributions into account. The most widely used method (Terrill and Melville 2000; Farmer et al. 1998) is that of Commander and McDonald (1991). Noting that Eq. (1) is a Fredholm integral of the first kind, they 
found a numerical solution to the integral by using a finite-element method, and then regularization was used to produce a stable result. This method is computationally expensive, since it requires an iterative procedure to smooth the result.

Caruthers et al. (1999) and Elmore and Caruthers (2003) proposed and developed a simpler alternative solution. They used the resonant approximation to produce a first-order solution and then made iterative corrections using the full forward attenuation calculation. A different numerical solution using both attenuation and phase velocity was developed by Duraiswamy et al. (1998). Choi and Yoon (2001) also proposed an iterative scheme using both the attenuation and the phase velocity to find a stable result.

In general, these methods are all computationally expensive and time consuming to carry out. In addition, these studies all have two things in common. First, they all set out to produce a general mathematical solution to Eq. (1), without considering the physical phenomena described by the kernel $\sigma$. Consideration of the physical meaning of that term and the shape of the function it describes leads to a solution specific to bubble inversion. Previous solution methods have been inefficient because they have all been very general mathematical treatments that would work for any kernel. Second, in the cases where an artificial bubble population was simulated and a full forward calculation of attenuation was carried out so that the inversion method could be tested, the issue of binning bubbles before the attenuation was calculated was not addressed, as far as the author is aware. During consideration of possible inversion methods for bubble measurements using an acoustical resonator, it became apparent that there is a simpler method for inversion, which takes account of the two points above and is considerably quicker to implement than previous methods. In addition, it removes some systematic errors that may have been overlooked in previous inversions.

\section{Method description}

As discussed above, the attenuation resulting from a single bubble resonance is significant over a finite frequency range, quantified by the bandwidth of the bubble resonance. Consequently, the attenuation measured at any single frequency is a sum of attenuation caused by bubbles with a resonant response close to the frequency of measurement and possibly also attenuation caused by geometric scattering from larger bubbles. Figure 2 demonstrates the problem for a flat bubble distribution (i.e., one for which there are equal numbers of bubbles in each radius increment). The acoustic cross section is shown with frequency for several bubbles at equal radius increments

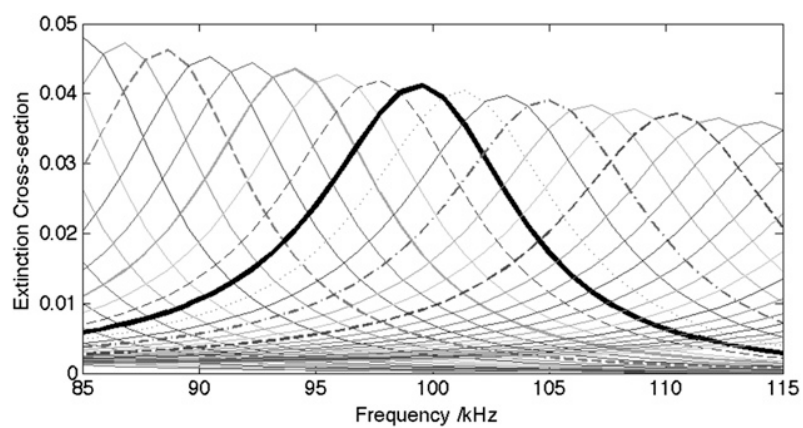

FIG. 2. A sample plot of the many individual cross sections that might add up to form the total attenuation. Most of the measured attenuation is due to bubbles that are not responding exactly at their resonance frequency, but resonate within approximately a bandwidth of the frequency of interest.

around $3 \mu \mathrm{m}$ in radius. The total measured attenuation will be proportional to the sum of all of these cross sections, and it can be seen that the resonance width is a crucial parameter in determining how much overlap there is between bubbles separated by a fixed radius increment. This resonant bandwidth depends on the damping associated with the resonance. For all the calculations described in this paper, the damping coefficients were calculated using Prosperetti's (1977) method. For bubbles with smaller radii (which resonate at higher frequencies), this resonant bandwidth is generally greater than that for larger bubbles. There is a small contribution to this bandwidth increase from the decrease in resonance quality factor as bubbles get smaller, but most of the bandwidth increase is just due to the definition of quality factor,

$$
Q=\frac{\omega_{0}}{\Delta \omega}
$$

where $Q$ is the bubble resonance quality factor, $\omega_{0}$ is its resonant angular frequency, and $\Delta \omega$ is the resonance bandwidth. As the resonant frequency increases, the bandwidth increases for the same quality factor. Figure 3 shows the normalized cross sections with frequency for four different bubble sizes. Each one is normalized to its maximum value.

Most attenuation measurements are equally spaced in frequency space, and it is evident from Fig. 3 that there are implications for the inversion if inversion points were chosen with this equal frequency spacing. An attenuation measurement at the lower frequencies would be influenced by only a small number of the bubbles in the corresponding bin, while at the higher frequencies the attenuation measurement would include a significant portion from bubbles in other bins. It seems that this approach could generate a systematic error. Most previous publications do not discuss the spacing of the inversion points used, but a large part of the effort that has been 


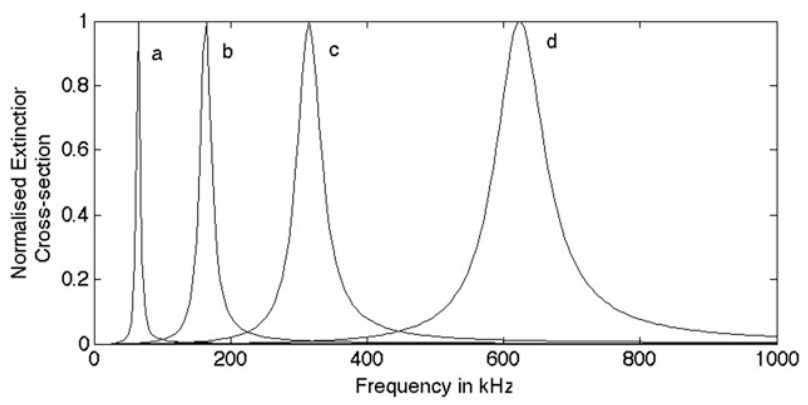

FIG. 3. The variation of extinction cross section with frequency for different bubble sizes, normalized to their maximum value. The bubble radii are (a) 50, (b) 20, (c) 10 , and (d) $5 \mu \mathrm{m}$.

expended on the inversion of Eq. (1) is compensating for this effect. It also seems to be the major reason for the failure of singular value decomposition inversion methods to produce stable solutions; if the inversion points are chosen with equal frequency spacing, then there are many possible combinations at the higher frequencies that can produce the same overall attenuation. This means that the matrix inversion does not have a unique solution and no stable result can be found.

The central theme of this paper is that the inversion accuracy and stability will be considerably improved if the spacing of the points used in the inversion is chosen so that the frequency bins each have a width in frequency space that matches the bandwidth of their central bubble. Figure 4 shows part of the frequency spectrum split up into bins with this spacing. Using this method, every bubble will be resonant in a bin (so the full forward attenuation calculation will include the correct number of bubbles), but the bin width reflects the frequency range that can be influenced by the bubbles inside that bin. This means that a matrix inversion method can be used, and that the solution will be smooth and stable because the mathematical construction reflects the physics of the process.

$$
\left[\begin{array}{ccc}
\sigma\left(a_{1}, f_{1}\right) & \sigma\left(a_{2}, f_{1}\right) & \sigma\left(a_{3}, f_{1}\right) \\
\sigma\left(a_{1}, f_{2}\right) & \sigma\left(a_{2}, f_{2}\right) & \sigma\left(a_{1}, f_{2}\right) \\
\sigma\left(a_{1}, f_{3}\right) & \sigma\left(a_{2}, f_{3}\right) & \sigma\left(a_{1}, f_{3}\right) \\
\cdots & &
\end{array}\right.
$$

where $f_{i}$ are the central frequencies of the frequency bins, and $a_{i}$ are the radii of the resonant bubbles at those frequencies. The values of $\alpha\left(f_{i}\right)$ are interpolated from the measured data points, and this matrix equation is solved using singular value decomposition to get the values $n\left(a_{i}\right)$.

The final stage is to divide the calculated $n\left(a_{i}\right)$ by the number of micron radius increments that are included in the corresponding frequency bin to get the bubble number per unit volume per micron radius increment.

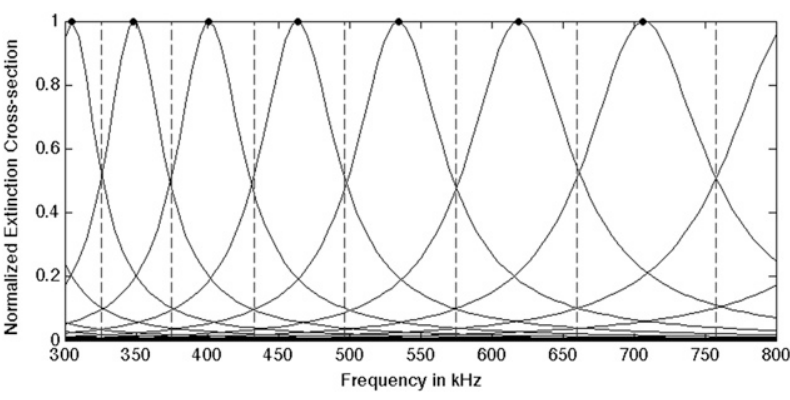

FIG. 4. A region of the spectrum showing the suggested frequency bin spacing and the cross section of the central bubble for each bin. Dotted vertical lines represent the frequency boundaries for the bins. The attenuation values used in the inversion will be at the frequency of the center of each bin (black filled circles), using interpolation between the measured acoustic data points.

The bandwidth of each bubble resonance can be derived by comparing Eq. (3) with Eq. (2); $1 / Q$ is equivalent to $2 \beta_{\text {tot }} / \omega_{0}$, so the width of each bin in hertz is given by

$$
\Delta f=\frac{\beta_{\text {tot }}}{\pi}
$$

Prosperetti's (1977) method is used to calculate the acoustic, thermal, and viscous effective viscosities $\mu$. Then, $\beta_{\text {tot }}$ was calculated using Eq. (5) as

$$
\beta_{\text {tot }}=\frac{2\left(\mu_{\text {viscous }}+\mu_{\text {acoustic }}+\mu_{\text {themal }}\right)}{\rho_{\text {water }} a_{0}^{2}},
$$

where $\rho_{\text {water }}$ is the density of the liquid and $a_{0}$ is the bubble radius.

Once the central bin frequencies are known, then a matrix of cross-sectional contributions can be calculated for these frequencies and the corresponding bubble radii by using Eq. (2). The resulting matrix equation is

$$
\cdots]\left[\begin{array}{c}
n\left(a_{1}\right) \\
n\left(a_{2}\right) \\
n\left(a_{3}\right) \\
\cdots
\end{array}\right]=\left[\begin{array}{c}
\alpha\left(f_{1}\right) \\
\alpha\left(f_{2}\right) \\
\alpha\left(f_{3}\right) \\
\cdots
\end{array}\right],
$$

\section{Method justification}

If there were no geometrical scattering by larger bubbles, this method would just consist of dividing the attenuation at the center frequency of each bin by the resonant cross section of the central bubble. The reason that a matrix formulation is needed is to take into account the geometrical scattering caused by bubbles larger than those in the bin of interest. Even though the matrix inversion is 


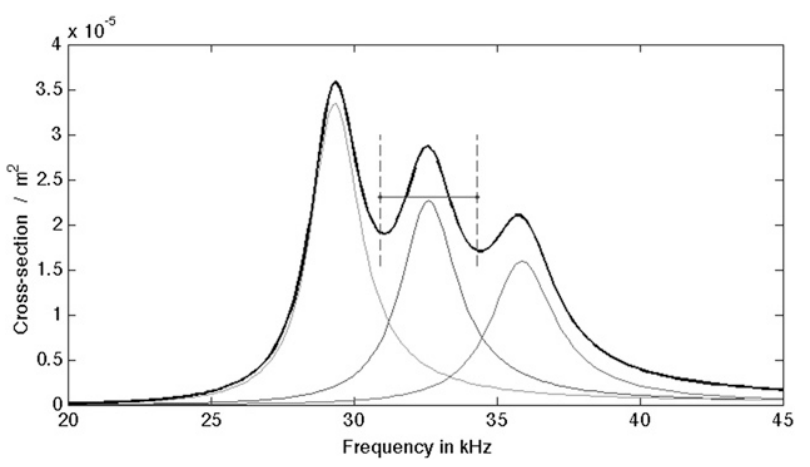

FIG. 5. The individual scattering cross sections associated with three bubbles (thin solid lines) are shown. The central bubble is the one of interest, and its bandwidth (vertical dotted lines), and therefore the width of the bin centered on this bubble, is shown. The bubbles on either side have resonant frequencies that are one bandwidth above and one bandwidth below this central bubble, and are therefore resonant at the middle of the bins on either side of the central bin shown here. The total attenuation from these three bubbles (thick black line) is shown. In between the vertical dotted lines there is a horizontal bar showing the average of the total attenuation over the frequency range between these lines, which is within $2 \%$ of the peak cross-sectional value for the single central bubble. If the central bubble is resonant at any frequency within the bandwidth shown, then the measured attenuation associated with it (measured at the center of the bin) could be any of the values shown between the vertical dotted lines. This is because any bubble that is off center in the central bin will have two corresponding bubbles that are off center in the bins on either side of the bin of interest. Averaging the total cross section over the frequency range (bracketed by the dotted lines) therefore produces the average attenuation associated with a bubble in that bin.

stable, we have not yet shown this will give the correct number of bubbles in the bin rather than a value that is proportional to the correct number. However, a simple physical argument presented in this section and the results in section 4 show that this is indeed the case. Consider a bubble in a frequency bin that is not necessarily resonant at the central frequency of that bin. There will also be attenuation at the measurement frequency from bubbles in the bins on either side, although their contribution will not dominate the measured attenuation. Let us suppose that for each bubble in a bin, we can find another bubble that is resonant at one bandwidth above, and a third that is resonant one bandwidth below the bubble of interest (so these two are contained within the bins on either side of the bin of interest), and that the influence of any other bubbles is small. We will call the bubble of interest here the "middle bubble" of the three, to distinguish it from the central bubble of the bin. It is a reasonable assumption for a continuous distribution that two such symmetrical corresponding bubbles can be found for any single middle bubble. Figure 5 shows this situation. The peak resonance of the middle bubble can be anywhere inside the bin. The average contribution that a bubble in this bin would make is therefore the mean value of the total attenuation at all

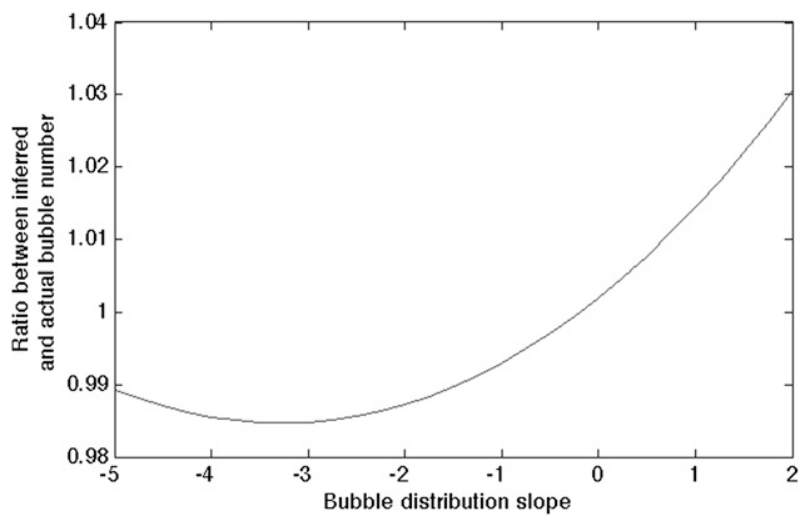

FIG. 6. The averaging of the total attenuation shown in Fig. 5 can be carried out for bubble size distributions with different slopes to see whether a sloped bubble distribution causes biases in the inversion result. For example, if the slope is -2 , then we expect more bubbles in the adjacent higher-frequency bin than in the adjacent lower-frequency bin. This would change the averaging depicted in Fig. 5, but we can compensate for that in the calculations, which is the situation shown here. This plot shows the ratio of total crosssectional average (the horizontal line in Fig. 5) and the actual crosssectional peak for different bubble size distribution slopes. Over the range of slopes we expect in oceanic bubble populations, the error is always less than $3 \%$.

the positions that correspond to the middle bubble being inside the bin. Figure 5 shows both the average value of the total attenuation over a full bandwidth centered on the middle bubble, and the value of the peak cross section of the middle bubble. In the case of a flat bubble distribution, the mean ratio of the average and the resonant cross section is 1.01. It is also possible to explore the effect of a bubble distribution slope (one in which the bubble number varies as $r^{x}$, where $x$ is a constant) by weighting the contribution of each bubble according to the number of bubbles expected with that distribution. Figure 6 shows the results for distributions with slopes between -5 and 2 , and the maximum error in this range generated is $3 \%$, which is insignificant on the scale of the uncertainties associated with bubble measurements using acoustic attenuation.

The meaning of these tests is that the effect of bubbles having noncentral positions within their frequency bin is approximately cancelled out by the influence of bubbles in the bins on either side, when the bins are each a bandwidth wide. An inversion carried out using the peak cross section of a central bubble in the bin will therefore produce the correct number of bubbles in that bin to within a few percent.

\section{Results}

This scheme was tested with a computational method designed to be as realistic as possible. Artificial bubble populations were generated containing between 20000 

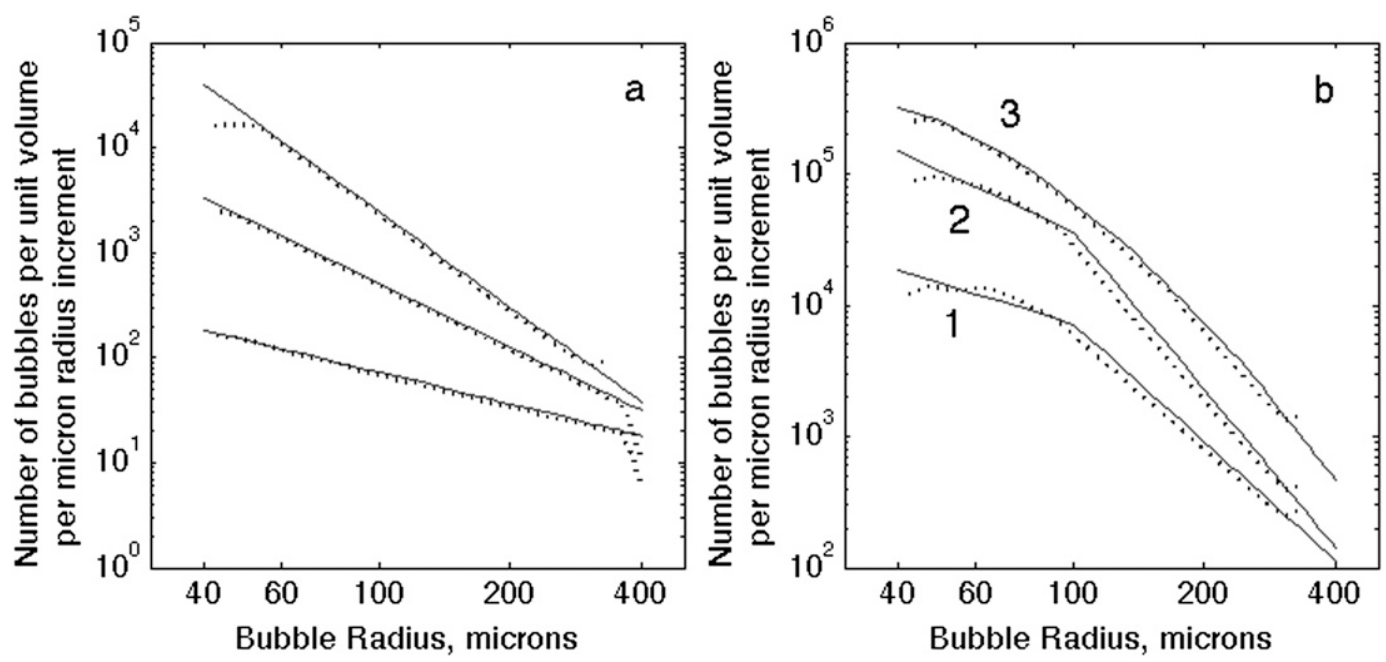

FIG. 7. Comparison of the initial bubble population distributions and the inverted distributions for a bubble population that only spans an order of magnitude in size. (a) The results for bubble populations with constant slopes of $-1,-2$, and -3 . The initial bubble population (thin solid line) is shown, and this was used to calculate acoustical attenuation with frequency, as described in the text. The bubble population calculated from that attenuation using the method described in this paper (dotted lines) is also shown. (b) Same as (a), but with information for more realistic distributions. Population 1 has a fixed slope of -3 for bubbles greater than $100-\mu \mathrm{m}$ radius and a slope of -1 for smaller bubbles. Population 2 has a slope of -4 for bubbles with radii above $100 \mu \mathrm{m}$ and -1.5 for smaller bubbles. Population 3 has a gradually changing slope, from -1 for the $40-\mu \mathrm{m}$ bubbles and -4 for the $400-\mu \mathrm{m}$ bubbles. Apart from the first and last points of any inversion, the method described in this paper produces results that are correct to within $10 \%$ in all cases, and much less for the distributions with a constant slope

and 200000 bubbles, with a distribution of sizes that fit the chosen bubble population distribution. The population was constructed by finding the number of bubbles required in each micron radius increment at radius $R$ in order to fit the distribution. A random number between 0 and 1 (designated $B$ ) was generated for each individual bubble, and that bubble was given a radius $r=R+B \Delta r$, where $\Delta r$ is $1 \mu \mathrm{m}$. Significantly, this meant that many different bubble radii were included within each frequency bin used for the inversion. "Measurement" frequencies were chosen, and the total attenuation was calculated at each chosen frequency by using the exact bubble radii for each bubble without any binning. This is equivalent to carrying out the sum represented by the integral in Eq. (1). This attenuation was then inverted using the method described here to test its accuracy.

Figure 7 shows the results for a radius range from 40$400 \mu \mathrm{m}$ for different bubble population slopes. For the results shown in Fig. 7, only bubbles that were resonant within the measurement frequency range were used to calculate the attenuation. The effect of bubbles outside the measurement frequency range will be discussed in section 5. Figure 7a shows that the inversion produced results accurate to within $2 \%$ over the whole radius range for a bubble size distribution with a constant slope. For more realistic populations with a varying slope shown in
Fig. 7b, the maximum deviation is just after a slope break, and then the maximum deviation is $10 \%$.

\section{Geometrical scatter}

The influence of the geometric scattering caused at higher frequencies by nonresonant larger bubbles could potentially become significant as the bubble measurement range is extended to higher frequencies and therefore smaller bubble sizes. We will consider two situations here. The first is the case where all the bubbles present are resonant within the attenuation measurement range, and it is examined here as context for the more important second situation. This second situation is where there are larger bubbles present that are resonant at frequencies below the measurement range. Because they cannot be measured using their resonance, there is the potential for the geometrical scattering that they cause within the measurement range to be interpreted as extra small bubbles. The calculations set out in the earlier part of this paper all include both resonant and geometrical scattering effects, but there is still the potential for error resulting from geometrical scattering effects of these bubbles, which are too large to be measured directly. We will use the methods outlined earlier in the paper to examine the potential magnitude of these errors. 

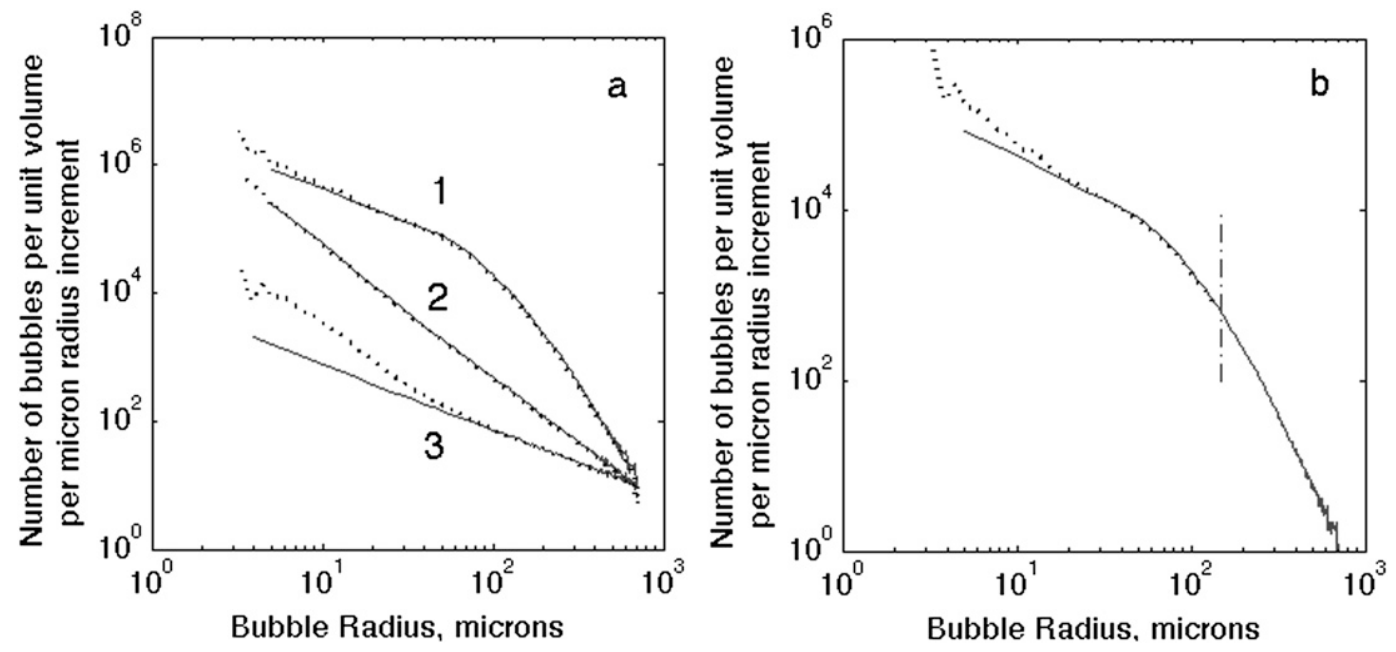

FIG. 8. Cases where geometric scatter may be more likely to influence the results, either from bubbles outside the measurement range or from larger bubbles within the measurement range that were slightly underestimated by the inversion. In all these cases, bubbles from 3 to $700 \mu \mathrm{m}$ were used to calculate the attenuation. (a) Population 2 has a fixed slope of -2 and population 1 has a more realistic shape, varying from a slope of -1 to a slope of -4 . In these two cases, the agreement between the original population and the inverted population is very good, within $3 \%$ at all radii. The lowest population has a slope of -1 , and a deviation between the original and inverted populations can be seen for radii less than $40 \mu \mathrm{m}$. (b) The curved population is the same as the uppermost one in (a), and the full attenuation was calculated from 5 to $1 \mathrm{MHz}$. However, only the attenuation values between 21 and $1 \mathrm{MHz}$ were used for the inversion, because this is the measurement range of the acoustical resonator that motivated this research. The size of bubble resonance at $21 \mathrm{kHz}$ (vertical dotted line), the lowest frequency within the measurement range, is shown. For the smallest bubble radii $(5 \mu \mathrm{m})$, the error is approximately a factor of 2 .

Figure 8 shows the actual and inverted bubble populations for different bubble size distributions with a radius range between 3 and $700 \mu \mathrm{m}$. For slopes less than -2 we do not expect the effects of geometric scatter to be significant, because the number of small bubbles is rising faster than the geometric contribution as bubble size decreases (as previously stated, the geometric contribution varies as $a_{0}^{2}$ ). Population 2 in Fig. 8 and populations with steeper slopes (not shown) bear out this expectation; if the slope is steeper than -2 for all the bubble sizes present, then geometrical scattering does not bias the results. However, for bubble distribution slopes greater than -2 , geometric scattering could cause errors at the smaller radii in this radius range. Population 3 in Fig. 8a has a slope of -1 , and the bias caused by geometrical scattering can be seen for bubble radii smaller than $40 \mu \mathrm{m}$. The cause of this deviation is likely to be the slight underestimation of the number of bubbles in the larger size classes measured. Undercounting these larger bubbles leaves some of the attenuation at higher frequencies unaccounted for, and the inversion will consequently overcount the number of smaller bubbles. This problem is almost entirely absent for slopes less than -2 , because smaller bubbles dominate the attenuation at their resonant frequencies, and small differences resulting from uncertainties in the larger bubble population are negligible by comparison. Slopes of approximately -1.5 have commonly been reported at the smaller radii (Deane and Stokes 2002; Farmer et al. 1998), and population 1 in Fig. 8a represents a more realistic ocean bubble size distribution. A small geometric bias can be seen for radii below $10 \mu \mathrm{m}$.

A situation of greater concern is shown in Fig. 8b, where there are significant numbers of bubbles present that are resonant at lower frequencies than the measurement range covers. Bubbles over the entire size range were used to calculate the attenuation, but the inversion was carried out using only the attenuation over a limited frequency range (equivalent to the resonant frequency range for $3-700-\mu \mathrm{m}$ bubbles). In this case, there is larger bias resulting from geometric scattering, up to a factor of 2 for $5-\mu \mathrm{m}$ radius bubbles, the smallest calculated here.

The results above show that geometric scatter only becomes a significant concern for measurements covering two orders of magnitude in the bubble radius in two situations. Problems may occur either when the bubble size distribution slope over the whole measurement range is greater than -2 or when there are large bubbles present that are resonant outside the frequency measurement range. The effect of these large bubbles is to generate "phantom bubbles," with a slope of approximately -2 at small bubble radii, and these can usually be identified by 
an inflection in the bubble slope at the radius at which the inverted population starts to differ from the actual bubble population. These indications may suggest, after the inversion has been done, that geometric scattering is a problem. No direct solution for this problem is offered here, although some straightforward modeling based on the individual populations measured could provide enough information to rule out geometrical scattering as a problem in some cases. For example, as shown in Fig. $8 \mathrm{~b}$, the population of larger bubbles that resonates outside the "measured" frequency range could reasonably have a slope between -3 and -5 . A simple forward calculation of the attenuation generated by the postulated large bubbles over the whole frequency range, followed by inversion using only the measurement frequency range, shows the number of phantom bubbles that the presence of larger bubbles could generate. An estimate of their importance can then be made. In the cases studied by the author using real bubble populations, the steepness of the bubble size distribution at the largest radii usually suggests that geometric scattering does not bias the inversion results. In practice, the tiny amount of attenuation caused by even a large number of bubbles less than $5 \mu \mathrm{m}$ in radius may be below the noise level of the detection hardware, and in our studies to date this instrument noise is the limitation on measurements of the smallest bubbles and not geometric scattering. However, for bubble populations that have slopes greater than -2 over a large size range, geometric scattering could be an insurmountable problem, and other techniques may be required to make simultaneous measurements in order to extend the bubble measurement range to smaller radii. The author suggests that the best approach may be to model the possible population of large bubbles for each individual bubble size distribution measurement in order to judge the extent to which geometric scattering could interfere with the results.

\section{Conclusions}

A simple and accurate method for inverting acoustic attenuation to infer the bubble population present in a liquid has been described. By spacing the inversion points so that each bubble bin has the same width in frequency space as the bandwidth of its central bubble, a simple matrix inversion can be used to infer the bubble population. Over a bubble radius range of an order of magnitude (with no bubbles present outside this range), this method is accurate to within $3 \%$. When there are bubbles present that are resonant outside the measurement frequency range, the simplicity of the method allows for a straightforward check for whether geometric scattering is likely to influence the final results.
Acknowledgments. This research was funded by the Office of Naval Research, Contract N00014-06-1-0072. The author is grateful to David Farmer for helpful discussions as the project progressed.

\section{APPENDIX}

\section{Damping Calculations}

The calculation of Eq. (2) is not straightforward for nonresonant bubbles because of the considerable potential for confusion about the correct damping coefficients to use. The method used for this study is outlined here, accompanied by a brief discussion of the most up-to-date literature on this topic and summary of the situations where these subtleties may produce significant changes to the overall result. This information is provided in an appendix because it does not affect the method described in this paper, although anyone using this method must carry out these calculations. The recent publications by Ainslie and Leighton $(2009,2011)$ have summarized some of the inconsistencies in the literature surrounding the damping calculations and provide advice on the most appropriate methods in different circumstances.

Damping for this study was calculated using the method of Prosperetti (1977), which leads directly to the values for $\beta_{\text {th }}, \beta_{\text {rad }}$, and $\beta_{\text {vis }}$ (i.e., the thermal, radiative, and viscous damping constants). These constants $\beta$ are the damping factors, defined by their use in the equation of motion for linear steady-state bubble oscillations,

$$
\ddot{a}+2 \beta_{\mathrm{tot}} \dot{a}+K\left(a-a_{0}\right)=F e^{i \omega t},
$$

where $K$ is the stiffness parameter, $F$ is the amplitude of the forcing at frequency $\omega$ over time $t, a$ is the bubble radius, and the dots denote time derivatives [see Ainslie and Leighton (2011), Eq. (62) for further details about these terms].

Here, $\beta_{\text {tot }}$ is the sum of the three $\beta$ terms; $\beta_{\text {tot }}$ is used directly in Eq. (2), and this is relatively straightforward. Table A1 shows the physical constants used for these calculations. The terms $\mu_{\mathrm{th}}, \mu_{\mathrm{rad}}$, and $\mu$ used in Eq. (5) of this paper are effective viscosities that Prosperetti (1977) uses in the calculation of $\beta$. The more complex part of the damping discussion revolves around the terms $d_{\mathrm{tot}}, d_{\mathrm{rad}}$, $d_{\mathrm{vis}}$, and $d_{\mathrm{th}}$. These are used in Eq. (2) only to scale the attenuation coefficient in order to convert it to an extinction coefficient.

For the calculations described in this paper, the $d$ terms were all calculated using Prosperetti's (1977) method, laid out in Eqs. (4.204)-(4.206) of Leighton (1994). These equations are not repeated here, because 
TABLE A1. List of parameter values used to calculate acoustic cross sections for the examples shown in this paper.

\begin{tabular}{|c|c|c|}
\hline Parameter, definition & Value & Units \\
\hline$\rho$, density of water & 1000 & $\mathrm{~kg} \mathrm{~m}^{-3}$ \\
\hline$\rho_{\mathrm{g}}$, density of air & 1.18 & $\mathrm{~kg} \mathrm{~m}^{-3}$ \\
\hline$\gamma$, the ratio of specific heat capacities of air & 1.4 & - \\
\hline$\mu$, viscosity of water & $8.9 \times 10^{-4}$ & Pa s \\
\hline$D_{g}$, thermal diffusivity of gas & $2.371 \times 10^{-5}$ & $\mathrm{~m}^{2} \mathrm{~s}^{-1}$ \\
\hline$\sigma$, surface tension of water & 0.07 & $\mathrm{~N} \mathrm{~m}^{-1}$ \\
\hline$D_{l}$, thermal diffusivity of water & $1.4 \times 10^{-7}$ & $\mathrm{~m}^{2} \mathrm{~s}^{-1}$ \\
\hline$k_{g}$, thermal conductivity of the gas & 0.024 & $\mathrm{~W} \mathrm{~m}{ }^{-1} \mathrm{~K}^{-1}$ \\
\hline$k_{l}$, thermal conductivity of water & 0.58 & $\mathrm{~W} \mathrm{~m}^{-1} \mathrm{~K}^{-1}$ \\
\hline$T$, temperature & 298 & $\mathrm{~K}$ \\
\hline$c$, speed of sound in water at $298 \mathrm{~K}$ & 1497 & $\mathrm{~m} \mathrm{~s}^{-1}$ \\
\hline$c_{g}$, speed of sound in air at $298 \mathrm{~K}$ & 345 & $\mathrm{~m} \mathrm{~s}^{-1}$ \\
\hline$c_{p, w}$, heat capacity of water & 4200 & $\mathrm{~J} \mathrm{~K}^{-1}$ \\
\hline$c_{p \text {,air }}$, heat capacity of air & 1012 & $\mathrm{~J} \mathrm{~K}^{-1}$ \\
\hline$M$, molar mass of gas & 0.029 & $\mathrm{~kg} \mathrm{~mol}^{-1}$ \\
\hline$p_{0}$, ambient pressure & $1 \times 10^{-5}$ & $\mathrm{~N} \mathrm{~m}^{-2}$ \\
\hline$R_{g}$, molar gas constant & 8.31 & $\mathrm{~J} \mathrm{~K}^{-1} \mathrm{~mol}^{-1}$ \\
\hline
\end{tabular}

very recent work has suggested that another approach is more appropriate, and repeating the equation risks propagating past errors in the literature. For the purposes of this paper, the approach used was sufficient to demonstrate the new technique described. The limitations of this simplification will now be described.

There are two issues: whether it is appropriate to use $d$ at all, and whether the available calculations are appropriate far from resonance. The first issue has been described in detail by Ainslie and Leighton (2011). They conclude that use of $d$ or $\delta$, the damping coefficient, is inappropriate in general because the choice of damping coefficient is dependent on the chosen definition for the resonance frequency and other parameters, and no entirely self-consistent set of definitions is available. This matters most far from resonance, so the well-known equations (as used in this paper) are still accurate for the consideration of bubbles close to resonance. However, on the basis of this new work, it seems that the most appropriate approach for the bubble size range discussed in this paper may be a modified one. This approach is laid out in Ainslie's paper in Eqs. (107) and (108) for larger bubbles where viscosity can be neglected, and in Eqs. (112) and (113) for smaller bubbles where the liquid can be considered incompressible. Future studies using a wide bubble size range should use these equations.

The second issue matters here because the matrix described by Eq. (6) in this paper could include the extreme case of large bubbles responding to high frequencies, that is, a $2-\mathrm{mm}$-radius bubble responding to a 300-kHz signal. Zhang and $\mathrm{Li}$ (2010) investigate the implications of a simplification in Prosperetti's (1977) method for bubbles far above their resonance frequency and define parameter ranges where it is appropriate.
That study suggests that for the range of bubble radii used in this paper, Prosperetti's method is appropriate, but just outside that range (e.g., a 2-mm bubble responding to $650-\mathrm{kHz}$ sound) it will start to fail.

The method of using broadband acoustic attenuation to measure a wide bubble size range is one of the few applications where these subtleties could become significant. Any future research into extending the measureable bubble size range beyond that used in this paper should take account of these complexities.

\section{REFERENCES}

Ainslie, M. A., and T. G. Leighton, 2009: Near-resonant bubble acoustic cross-section corrections, including examples from oceanography, volcanology and biomedical ultrasound. J. Acoust. Soc. Amer., 126, 2163-2175.

—, and —, 2011: Review of scattering and extinction crosssections, damping factors and resonance frequencies of a spherical gas bubble. J. Acoust. Soc. Amer., 130, 3184-3208.

Breitz, N., and H. Medwin, 1989: Instrumentation for in situ acoustical measurements of bubble spectra under breaking waves. J. Acoust. Soc. Amer., 86, 739-743.

Caruthers, J. W., P. A. Elmore, J. C. Novarini, and R. R. Goodman, 1999: An iterative approach for approximating bubble distributions from attenuation measurements. J. Acoust. Soc. Amer., 106, 185-189.

Choi, B. K., and S. W. Yoon, 2001: Acoustic bubble counting technique using sound speed extracted from sound attenuation. IEEE J. Oceanic Eng., 26, 125-130.

Commander, K., and E. Moritz, 1989: Off-resonant contributions to acoustical bubble spectra. J. Acoust. Soc. Amer., 85, 2665-2669. and R. J. McDonald, 1991: Finite element solution of the inverse problem in bubble swarm acoustics. J. Acoust. Soc. Amer., 89, 592-597.

Deane, G. B., and M. D. Stokes, 2002: Scale dependence of bubble creation mechanisms in breaking waves. Nature, $\mathbf{4 1 8}$, 839-844. 
Duraiswamy, R., S. Prabhukumar, and G. L. Chahine, 1998: Bubble counting using an inverse acoustic scattering method. J. Acoust. Soc. Amer., 104, 2700-2017.

Elmore, P. A., and J. W. Caruthers, 2003: Higher-order corrections to an iterative approach for approximating bubble distributions from attenuation measurements. IEEE J. Oceanic Eng., 28, 117-120.

Farmer, D. M., S. Vagle, and A. D. Booth, 1998: A free-flooding resonator for measurement of bubble size distributions. J. Atmos. Oceanic Technol., 15, 1132-1146.

Leighton, T. G., 1994: The Acoustic Bubble. Academic Press, 296 pp.

Medwin, H., 1970: In situ acoustic measurements of bubble populations in coastal ocean waters. J. Geophys. Res., 75 (3), 599-611.
1977: Acoustical determinations of bubble size spectra. J. Acoust. Soc. Amer., 62, 1041-1044.

_ 2005: Sounds in the Sea. Cambridge University Press, 196 pp. Prosperetti, A., 1977: Thermal effects and damping mechanisms in the forced radial oscillations of gas bubbles in liquids. J. Acoust. Soc. Amer., 61, 17-27.

Terrill, E. J., and W. K. Melville, 2000: A broadband acoustic technique for measuring bubble size distributions: Laboratory and shallow water measurements. J. Atmos. Oceanic Technol., 17, 220-239.

Zhang, Y., and S. C. Li, 2010: Notes on radial oscillations of gas bubbles in liquids: Thermal effects. J. Acoust. Soc. Amer., 128, EL306-EL309. 